\title{
Preparation, Structure, and Properties of Polystyrene-Microsphere-Reinforced PEG-Based Hydrogels
}

\author{
Chen Zhang, Zhanping Zhang * and Yuhong Qi
}

check for

updates

Citation: Zhang, C.; Zhang, Z.; Qi, Y Preparation, Structure, and Properties of Polystyrene-Microsphere-Reinforced PEG-Based Hydrogels. Polymers 2021 13, 2605. https://doi.org/10.3390/ polym13162605

Academic Editors: Miloslav Pekař, Anton Blencowe and Elisabetta Ranucci

Received: 4 June 2021

Accepted: 3 August 2021

Published: 5 August 202

Publisher's Note: MDPI stays neutral with regard to jurisdictional claims in published maps and institutional affiliations.

Copyright: (c) 2021 by the authors. Licensee MDPI, Basel, Switzerland. This article is an open access article distributed under the terms and conditions of the Creative Commons Attribution (CC BY) license (https:// creativecommons.org/licenses/by/ $4.0 /)$.
Department of Materials Science and Engineering, Dalian Maritime University, Dalian 116026, China; pudding@dlmu.edu.cn (C.Z.); yuhong_qi@dlmu.edu.cn (Y.Q.)

* Correspondence: zzp@dlmu.edu.cn

Keywords: polystyrene microspheres; polyethylene glycol; composite hydrogel; structure; property

\section{Introduction}

Hydrogels are a gel system formed by hydrophilic polymers and water molecules with a three-dimensional interconnection network structure that can swell in water but not dissolve [1-3]. In 1960, Wichterle and Lim [4] found a soft, swelling, elastic and transparent material in the presence of a small number of crosslinking agent, and a certain number of water or an appropriate solvent during the polymerization of hydroxyethyl methacrylate monomer. This research result opened a prelude to the synthesis and application of hydrogels. Subsequently, the diverse of hydrogels emerged and developed rapidly. The hydrogels are widely used in tissue engineering [5-7], biomedical devices [8,9], microfluidic, optical actuators [10,11], and the marine industry [12,13] due to its high moisture, physiological fluid, and high elasticity. From the source of preparing hydrogels, they can be divided into natural macromolecule hydrogels and synthetic hydrogels; according to the different ways of crosslinking, they can be divided into physically cross-linked hydrogels and chemical cross-linked hydrogels; according to different sizes of hydrogels, they can be divided into macroscopic hydrogels, micron hydrogels, nano hydrogels, etc. [14].

It is a common phenomenon that the swelling property of hydrogels is accompanied by complex water evaporation and water absorption in the actual environment, which will lead to the weakening of hydrogels and loss of their original properties, such as mechanical properties. On the one hand, by grafting hydrogels onto carriers with high mechanical strength, the mechanical properties of hydrogels can be greatly improved. On the other hand, functionalized hydrogels can be obtained by grafting certain functional chains onto hydrogels. Ewaramm [15] copolymerized N-vinyl caprolactam onto guar gum, then it mixed with sodium alginate and cross-linked with glutaraldehyde to obtain a composite hydrogel, and applied the hydrogel to control release, which can effectively control the release of zidovudine (a drug for the treatment of AIDS) through $\mathrm{pH}$ and temperature. 
Zhuo [16] copolymer P(HEMA-co-MMA) obtained by copolymerization of Hydroxyethyl methacrylate (HEMA) with methyl methacrylate (MMA) can further improve the mechanical strength of hydrogels while maintaining the hydrophilic properties. Li [17] prepared a novel PAM-GO nanocomposite hydrogel using graphite oxide (GO) as a multifunctional crosslinking agent and acrylamide (AM) as a monomer. Compared with the traditional PAM hydrogel, the obtained hydrogel has excellent mechanical properties, elongation at break is more than $3000 \%$, tensile strength up to $385 \mathrm{kPa}$, and excellent swelling property. Zhu $[18,19]$ used photoactive polystyrene (PS) nanoparticles as a crosslinking agent to prepare a covalent crosslinking PAM-PS nanocomposite hydrogel with excellent mechanical properties and stability. The mechanical properties and swelling degree of the gel changed with the number of PS microspheres and monomers in the initial reaction solution. In addition to the interactions between the polymer and PS microspheres, hydrogen bonding between the polymer chains also plays an important role in gel formation.

Because there may be grafting, interpenetration, or ionic bonding between the core and shell of core-shell polymer microspheres, the wear resistance, water resistance, weather resistance, pollution resistance, radiation resistance, and tensile strength of the polymer can be significantly improved, and the film-forming temperature can be reduced and the processing property can be improved as well [20]. Similarly, core-shell microgels are special gels with a special structure formed by coating the gel shell layer on the gel core. Such gel particles have core and shell properties due to their composition and structure characteristics [21,22]. During preparation, according to the properties of the monomers and the feeding sequence, the microgels with normal core-shell structure or special-shaped core-shell structure can be formed [23,24]. Pelton [25] reported for the first time the preparation and characterization of a thermo-sensitive poly ( $\mathrm{N}$-isopropylacrylamide), namely PNIPAM microgel. They first used soap-free emulsion polymerization to prepare polystyrene (PS) as the core and PNIPAM as the shell hydrogel particles. Later, many people reported the preparation of PNIPAM nanohydrogels and copolymerized hydrogels containing PNIPAM blocks by different and similar methods [26,27]. Polyethylene glycol (PEG) is a kind of polymer with $-\mathrm{CH}_{2} \mathrm{CH}_{2} \mathrm{O}$ - as the structural unit. In its main chain structure, the oxygen atom has a high polarity, so this polymer has a high hydrophilicity. At present, the main chemical steps of preparing PEG-based hydrogels are to modify the hydroxyl terminal of PEG and introduce some controllable chemical crosslinking functional groups, gelation is then realized [28]. For example, ethylene is modified on the two ends of PEG by the esterification method, and the gel can be formed by olefin addition of mercapto group reaction in the presence of polymercaptan [29].

Due to the characteristics of simple preparation and excellent performance of nanocomposite hydrogels, polymer hydrogels are irreplaceable in many fields. However, at the present stage, the properties of polymer hydrogels also have some defects, such as poor mechanical properties and single function, which hinder their application to a certain extent. Therefore, the research on nanocomposite hydrogels will continue and open up a broader field of application.

To improve the mechanical properties of the hydrogel, novel nanocomposite hydrogels were prepared in this paper, which was designed that PEG hydrogel as a base and nano microspheres were used to reinforce PEG hydrogel. PEG-based nanospheres composite hydrogels were prepared by two-step method. Styrene (St), hydroxyethyl methacrylate (HEMA), and polyethylene glycol methyl ether methacrylate (PEGMA) were used as monomers to prepare nanometer microspheres with core-shell structures by soapfree emulsion polymerization of styrene. The chemical structure, morphology, swelling property, contact angle, surface energy, and mechanical compression property of PS-PEG hydrogels were tested and characterized to prepare nano-composite hydrogels with high compressive strength. 


\section{Materials and Methods}

\subsection{Materials}

The reagent used in this study was styrene (St, CP), purchased from Tianjin Damao Chemical Reagent Co., Ltd. (Tianjin, China). Hydroxyethyl methacrylate (HEMA, AR), polyethylene glycol methyl ether methacrylate (PEGMA, AR), azobisisobutamidine hydrochloride (AIBA, AR), isphorone diisocyanate (IPDI, AR), 1, 4-butanediol (BDO, AR) and polyethylene glycol (PEG) with an average molecular weight of 2000 were purchased from Aladin Biochemical Technology Co., Ltd. (Shanghai, China). Polyether triol (HSH330, CP) was purchased from Jiangsu Hai'an Petrochemical Plant (Hai'an, China). Xylene (AR) was purchased from Tianjin Tianli Chemical Reagent Co., Ltd. (Tianjin, China). Ethyl acetate $(\mathrm{AR})$, potassium bromide (KBr, AR), and cyclohexanone (AR) were purchased from Tianjin Kemiou Chemical Reagent Co., Ltd. (Tianjin, China).

\subsection{Synthesis Principle and Process Method}

\subsubsection{Synthesis Principle}

1. Chemical Reaction Principle of Preparing Polystyrene Microspheres

Polystyrene microspheres were prepared by soap-free emulsion polymerization using styrene, HEMA and PEGMA as reaction monomers and AIBA as initiator, as showed in Figure 1.

The chemical reaction principle of preparing PS microspheres

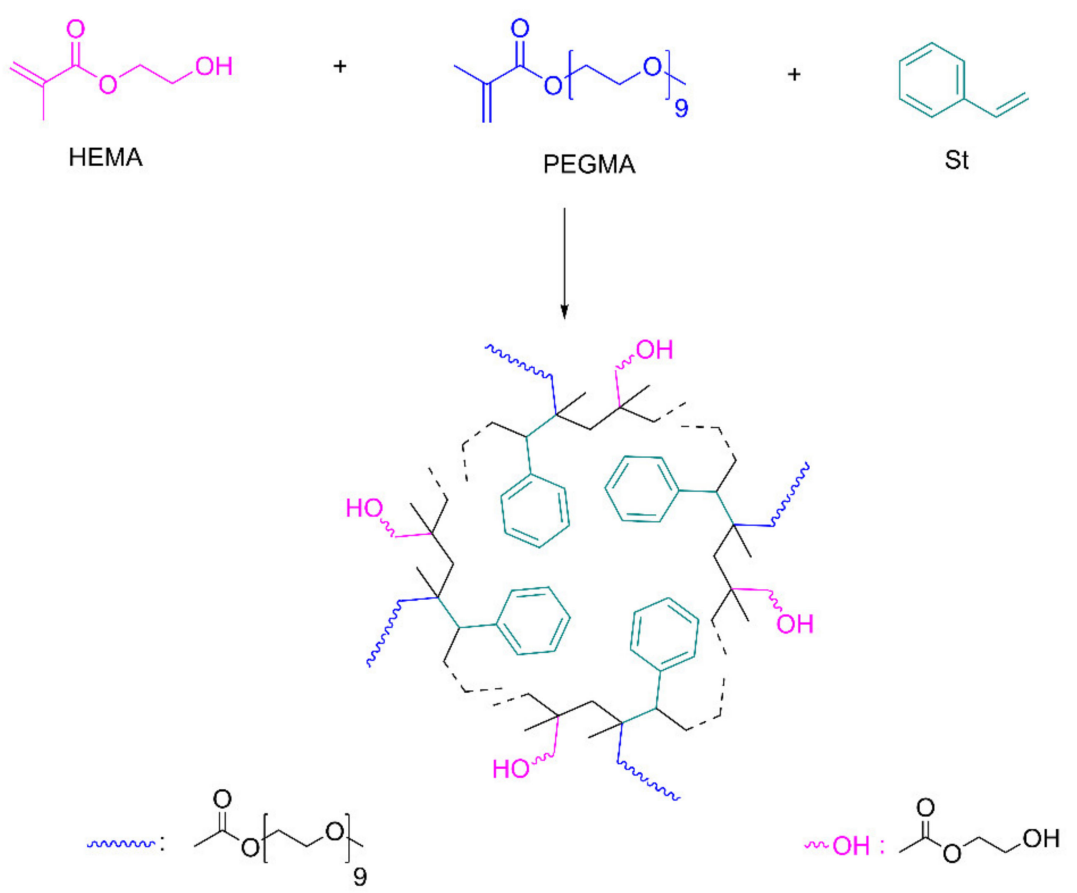

PS Microspheres

Figure 1. Chemical reaction principle of preparing PS microspheres.

2. Chemical Reaction Principle of Preparation of PS-NCO Intermediates

PEG2000 and IPDI were used as the reactive monomers to conduct the initial reaction in the mixed solvent. Then the PS microspheres powder prepared in the previous step was added into the system, the -NCO in excess IPDI will react with the -OH on the surface of PS microspheres to obtain PS-NCO intermediates through in-situ polymerization, as shown in Figure 2. 


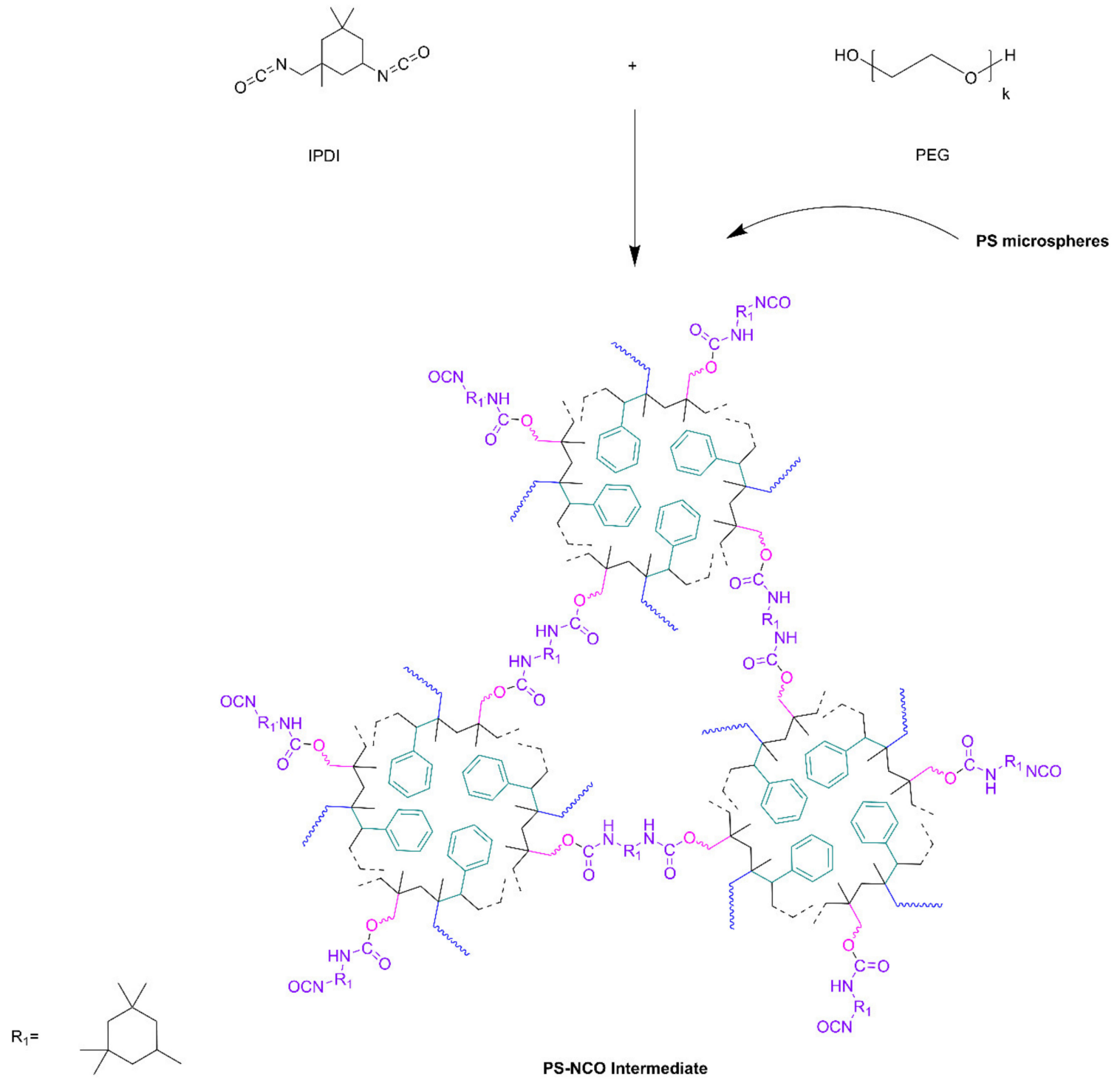

Figure 2. Chemical reaction principle of preparing PS-NCO.

3. Chemical Reaction Principle of Preparation of PS-PEG Hydrogel

Using PEG2000, IPDI, and HSH330 as monomers, the isocyanate of IPDI was firstly reacted with the hydroxyl terminations of PEG2000 and HSH330 by solution polymerization method. Then PS-NCO and BDO was added into the reaction system and finally, PS-PEG hydrogel was obtained, as showed in Figure 3. 

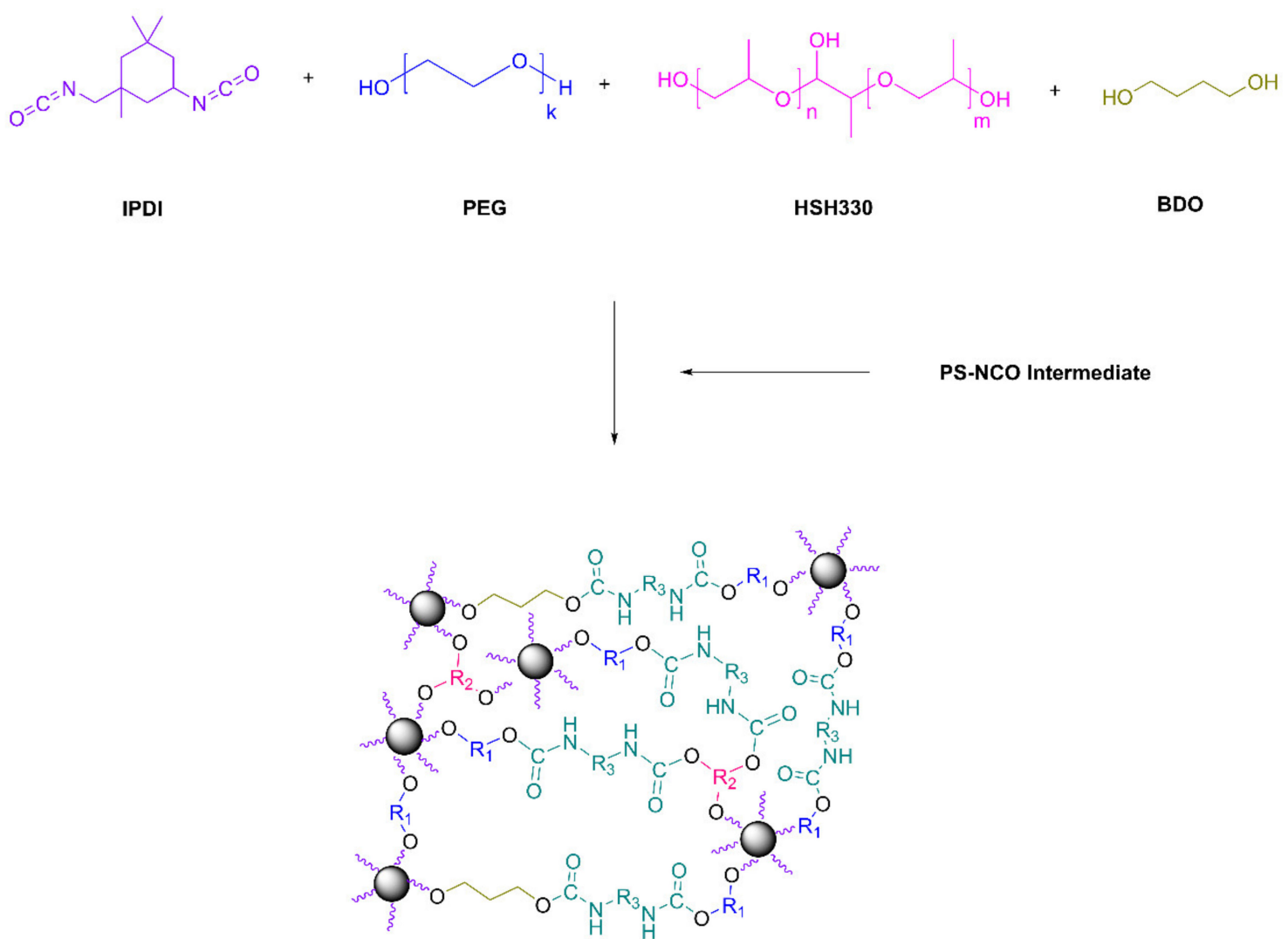

PS-NCO Intermediate

PS-PEG hydrogel

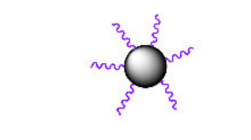

PS-NCO Intermediate

$$
\mathrm{HO}^{-\mathrm{R}_{1} \mathrm{OH}}
$$

PEG

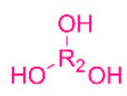

HSH330

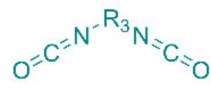

IPDI

Figure 3. Chemical reaction principle of preparing PS-PEG hydrogel.

The monomer mass of PS microspheres was $7.1 \mathrm{wt} \%, 14.2 \mathrm{wt} \%, 21.3 \mathrm{wt} \%$, and $28.4 \mathrm{wt} \%$. The corresponding products were named as PS7-PEG, PS14-PEG, PS21-PEG, and PS28-PEG. The sample without PS microspheres is labeled as PS0-PEG.

\subsubsection{Technological Process}

The first step of the synthesis, A $500 \mathrm{~mL}$ four-neck flask was connected to a SZCL-2A digital intelligent control magnetic stirrer (Yuhua Instrument Co., Ltd., Gongyi, China), and $4 \mathrm{~g}$ HEMA, $6 \mathrm{~g}$ PEGMA, and $250 \mathrm{~g}$ deionized water were added. Meanwhile, $20 \mathrm{~g}$ styrene was put into $50 \mathrm{~mL}$ separating funnel, and control in all added into the flask about 30 min. Used DW-3 high-speed digital display electric stirrer (Yuhua Instrument Co., Ltd., Gongyi, China), and pre-emulsified at $3000 \mathrm{rpm}$ for $1 \mathrm{~h}$. Under the protection for nitrogen, the temperature was raised to $60^{\circ} \mathrm{C}$ by using DF-101Sheat-collecting thermostatic heating magnetic stirrer (Yuhua Instrument Co., Ltd., Gongyi, China). In addition, $0.5 \mathrm{~g}$ AIBA was dissolved in $20 \mathrm{~g}$ deionized water, put into a $50 \mathrm{~mL}$ separatory funnel, and control about $1 \mathrm{~h}$ to all drip into the flask for reaction. After the content was completed, the reaction was carried out for $8 \mathrm{~h}$ with $280 \mathrm{rpm}$. Then, 200 mesh gauze was used to filter out the 
larger particles and used LC-10A-50N vacuum freeze-drying (Lichen Bangxi Instrument Technology Co., Ltd., Shanghai, China) is performed (Freeze for $12 \mathrm{~h}$, dry for $24 \mathrm{~h}$ ). Then, the powder was washed with added ethyl acetate and deionized water successively in a mass ratio of 1:1, used KQ-300E ultrasonic cleaner (Ultrasonic Instrument Co., Ltd., Kunshan, China) to wash for $40 \mathrm{~min}$, and TDL-50C centrifuge (Anting Scientific Instruments Co., Ltd., Shanghai, China) was used to remove the supernatant by centrifugation for $15 \mathrm{~min}$ with $4000 \mathrm{rpm}$. Repeat three times to wash away the unreacted monomers. After finishing freeze-drying again (Freeze for $12 \mathrm{~h}$, dry for $24 \mathrm{~h}$ ), the powder obtained is ground and sealed, and the yield of PS microsphere powder prepared at one time was about $26 \mathrm{~g}$. The specific process flow is shown in Figure 4a.

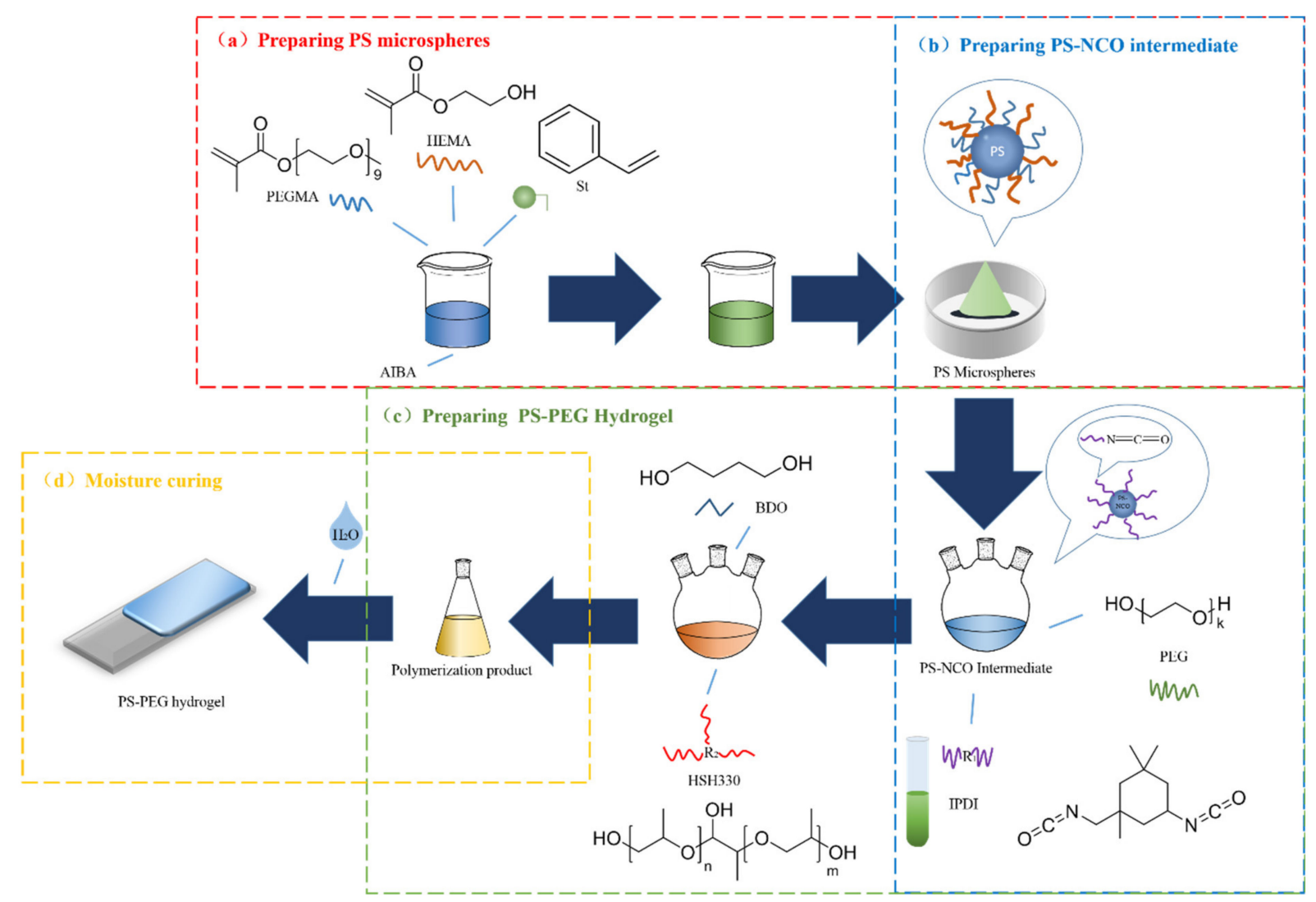

Figure 4. Preparation process of PS-PEG hydrogel (a) PS microspheres; (b) PS-NCO intermediate; (c) PS-PEG hydrogel; (d) Moisture curing.

The next stage of the work, $44 \mathrm{~g}$ PEG was dried under reduced pressure at $120{ }^{\circ} \mathrm{C}$ for $3 \mathrm{~h}$ to remove water and dissolved gases. The mixed solvent (Xylene: Ethyl acetate: Cyclohexanone $=2: 2: 1$ ) was filled, mixed, and stirred for $30 \mathrm{~min}$, and then the temperature was raised to $70{ }^{\circ} \mathrm{C}$. $10 \mathrm{~g}$ IPDI was added to the flask, reacted for $3 \mathrm{~h}$, and then the temperature was lowered to $60^{\circ} \mathrm{C}$, the $10 \mathrm{~g}$ PS microspheres powder was slowly added, isocyanate terminated PS-NCO intermediate is obtained after $5 \mathrm{~h}$, which is encapsulated and ready for use. The specific process flow is showed in Figure $4 \mathrm{~b}$.

Finally, $40 \mathrm{~g}$ PEG and $10 \mathrm{~g}$ HSH330 were dried under reduced pressure at $120{ }^{\circ} \mathrm{C}$ for $3 \mathrm{~h}$, mixed with $80 \mathrm{~g}$ solvent and stirred for $30 \mathrm{~min} .10 \mathrm{~g}$ IPDI was added to the flask at $70{ }^{\circ} \mathrm{C}$ and reacted for $3 \mathrm{~h}$. When temperature cooled to $60^{\circ} \mathrm{C}, 10 \mathrm{~g}$ PS-NCO solution was slowly added to the flask. After adding the chain extender $0.5 \mathrm{~g} \mathrm{BDO}$, the reaction ends after $5 \mathrm{~h}$. The yield of PS-PEG hydrogel was about $160 \mathrm{~g}$. The specific process flow is showed in Figure 4c. When it is lowered to room temperature, pour it into a polytetrafluoroethylene board for casting, and put in GDHS-2005A constant temperature and humidity box (Jinghong Experimental Equipment Co., Ltd., Shanghai, China), adjust 
humidity $70 \%$ RH to wet solidify to get PS-PEG hydrogel. The specific process flow is showed in Figure $4 \mathrm{~d}$.

\subsubsection{Preparation of Samples}

The part of PS-PEG hydrogel solution was painted on glass slides whose dimensions are $76.2 \mathrm{~mm} \times 25.4 \mathrm{~mm} \times 1 \mathrm{~mm}$, the rest was poured into a Teflon mold whose dimensions are $20 \mathrm{~mm} \times 20 \mathrm{~mm} \times 9 \mathrm{~mm}$. The specimens were placed in a dust-free room temperature environment of $70 \% \mathrm{RH}$, cured for 7 days. The former cured was used to characterize the chemical structure and surface morphology, the latter cured to measure contact angle, swelling and compressive properties.

\subsection{Characterizations}

\subsubsection{Chemical Structure}

Fourier Transform Infrared Spectrometer (FTIR)

FTIR (EQUINOX5, Bruker, Karlsruhe, Germany) was used to analyze the chemical structure of the PS microspheres with $\mathrm{KBr}$ tablet method. $200 \mathrm{mg} \mathrm{KBr}$ and $2 \mathrm{mg}$ PS microsphere powder were mixed and ground in an agate mortar with $\varphi 60 \mathrm{~mm}$ for $1 \mathrm{~min}$, and then, put into the tableting mold with $\varphi 13 \mathrm{~mm}$, pressurized to $20 \mathrm{MPa}$, and stayed for $2 \mathrm{~min}$. After that, take out the mold and put the tablet into the tablet holder for infrared test. The scanning range was 4000 to $400 \mathrm{~cm}^{-1}$, the resolution was $2 \mathrm{~cm}^{-1}$, and the number of scans was 32 times.

FTIR (PERKINELMER, Waltham, MA, USA) was used to analyze the chemical structure of the PS-NCO intermediate and PS-PEG hydrogels with attenuated total reflection mode, the scanning range was 4000 to $650 \mathrm{~cm}^{-1}$, the resolution was $2 \mathrm{~cm}^{-1}$, and the number of scans was 32 times.

\section{X-ray Diffraction $(\mathrm{XRD})$}

XRD spectrum of the samples was performed with D/MAX-Ultima X-ray diffractometer (Rigaku Denki, Tokyo, Japan). The instrument used copper-palladium ceramics with the test range was $10-90^{\circ}$, the step size was $0.02^{\circ}$, and the time per step was set at $8^{\circ} \mathrm{min}^{-1}$.

\subsubsection{Surface Morphology}

Scanning Electron Microscope (SEM)

The morphology of the samples was observed by Supra-55-sapphire SEM (Carl Zeiss AG, Jena, Germany). Take a square monocrystalline silicon wafer with a side length of $2 \mathrm{~cm}$, and the PS microspheres emulsion was diluted and then dropped on the wafer. After being dried, liquid conductive adhesive drops are used to seal the edges around the wafers, and then the microstructure characteristics were observed by SEM. In addition, the hydrogel was prepared into a sheet of $10 \mathrm{~mm} \times 10 \mathrm{~mm} \times 1 \mathrm{~mm}$, and adhered to the surface of the metal block with conductive tape. The JFC-1100 ion sputter (Japan Electronics Ltd., Tokyo, Japan) used direct current, adjusted the voltage and current to $5 \mathrm{kV}$ and $5 \mathrm{~mA}$, respectively, and sputtering on the surface of the specimen for $2 \mathrm{~min}$. The observation mode is SE2, and the acceleration voltage is $1 \mathrm{kV}$.

\section{Transmission Electron Microscope (TEM)}

JEOL-2100 TEM (Japan Electronics Ltd., Tokyo, Japan) was used to observe the morphology of nano microspheres. After diluting the emulsion in absolute ethanol, it was ultrasonically dispersed for $15 \mathrm{~min}$, then dripped onto a copper net for air-drying, and the accelerating voltage was $200 \mathrm{kV}$.

\section{Confocal Laser Scanning Microscope (CLSM)}

OLS4000 CLSM (Olympus, Tokyo, Japan) was used to observe and analyze the surface and fracture of the PS-PEG hydrogels. The surface roughness (Sa) of the samples were analyzed by LEXT analysis software. 


\subsubsection{Physical Properties}

Differential Scanning Calorimetry (DSC)

DSC measurement was performed on NETZSCH DSC 200F3 differential scanning calorimeter (NETZSCH-Gerätebau $\mathrm{GmbH}$, Selb, Germany) under a nitrogen flow of $5{ }^{\circ} \mathrm{C}$ $\mathrm{min}^{-1}$. The hydrogel was dried for $48 \mathrm{~h}$ and put into an aluminum crucible after removing the water. The scanning temperature range was set at $-100-200{ }^{\circ} \mathrm{C}$.

\section{Swelling Properties}

The composite hydrogel obtained after curing for 7 days were cut into cuboid specimens whose dimensions are $2.5 \mathrm{~mm} \times 1 \mathrm{~mm} \times 0.5 \mathrm{~mm}$, and immersed in deionized water for $48 \mathrm{~h}$. The samples were taken out at different time intervals, quickly dry the water on the sample surface with a filter paper, and put it into a precision balance for weighing. The swelling degree of the hydrogel SD = (WS $-\mathrm{W} 0) / \mathrm{W} 0$. WS and W0 are respectively the mass of the hydrogel when swelling to a certain time and primitive hydrogel.

\section{Contact Angle (CA) and Surface Free Energy}

$\mathrm{CA}$ of the hydrogels were measured by hanging drop method at room temperature using JC2000C Contact Angle Measurement Instrument (Zhongchen Digital Technology Equipment Co., Ltd., Shanghai, China), and used a syringe to drop $3 \mu \mathrm{L}$ of the liquid (deionized water or diiodomethane) onto the surface of the sample. Meanwhile, calculating for $5 \mathrm{~min}$ for dynamic water contact angle (DWCA) and dynamic diiodomethane contact angle (DDCA). Owens two-liquid method [30] was used to calculate the surface free energy of PS-PEG hydrogel coating by calculating DWCA and DDCA.

\subsubsection{Mechanical Properties}

According to GB/T 1041-92, the hydrogel samples wet-cured for 7 days were made into cuboid mold with a length of $20 \mathrm{~mm}$, a width of $20 \mathrm{~mm}$ and a height of $9 \mathrm{~mm}$. The compression performance of hydrogels was tested by UTM 5105 computer-controlled electronic universal testing machine (Jinan Wance Electrical Equipment Co., Ltd., Jinan, China). The hydrogel sample was placed in the middle of the test bench and compressed at a speed of $1 \mathrm{~mm} / \mathrm{min}$. The elastic modulus was calculated according to the slope of the strain within $0.1 \mathrm{~mm} / \mathrm{mm}$ in the stress-strain curve.

\section{Results}

\subsection{Chemical Structure and Morphology of PS Microspheres}

The PS microspheres powder prepared by the freeze-drying method was tested by FTIR, as showed in Figure 5, indicating that the absorption peak at $3435 \mathrm{~cm}^{-1}$ was the vibration absorption peak of the hydroxyl group. The stretching vibration absorption peaks at $3000-3100 \mathrm{~cm}^{-1}$ were $\mathrm{C}-\mathrm{H}$ bond of benzene ring. The absorption peaks at $1724 \mathrm{~cm}^{-1}$, $1801 \mathrm{~cm}^{-1}, 1870 \mathrm{~cm}^{-1}$, and $1945 \mathrm{~cm}^{-1}$ were weak bands unique to PS, corresponding to the frequency double and group frequency absorption peaks of the out-of-plane bending vibration of the aromatic ring C-H. While the strong absorption peak at $1633 \mathrm{~cm}^{-1}$ was the stretching vibration absorption peak of the carbonyl group on the ester group, its original absorption peak was $1740 \mathrm{~cm}^{-1}$. Because of the high polarity of carbonyl group, it can form conjugate effect with the benzene ring, so that the absorption peak of carbonyl group moves to the direction of low wave number, that is, the absorption frequency decreases [31]. The absorption peaks at $1450 \mathrm{~cm}^{-1}, 1494 \mathrm{~cm}^{-1}$, and $1600 \mathrm{~cm}^{-1}$ are the skeleton vibration absorption peaks of the benzene ring. The absorption peaks at $1354 \mathrm{~cm}^{-1}$ and $1382 \mathrm{~cm}^{-1}$ were the in-plane bending vibration absorption peaks of alkyl C- $\mathrm{H}$, and the absorption peak of ether bond at $1101 \mathrm{~cm}^{-1}$. In the meanwhile, the absorption peaks at $842 \mathrm{~cm}^{-1}, 906 \mathrm{~cm}^{-1}$, and $945 \mathrm{~cm}^{-1}$ were the $\mathrm{C}-\mathrm{H}$ out-of-plane flexural vibration absorption peaks of the benzene ring, and the absorption peaks at $700 \mathrm{~cm}^{-1}$ and $759 \mathrm{~cm}^{-1}$ were the monosubstituted benzene ring. The above characteristic peaks indicate that PS microspheres have hydroxyl, carbonyl, and benzene ring functional groups. 


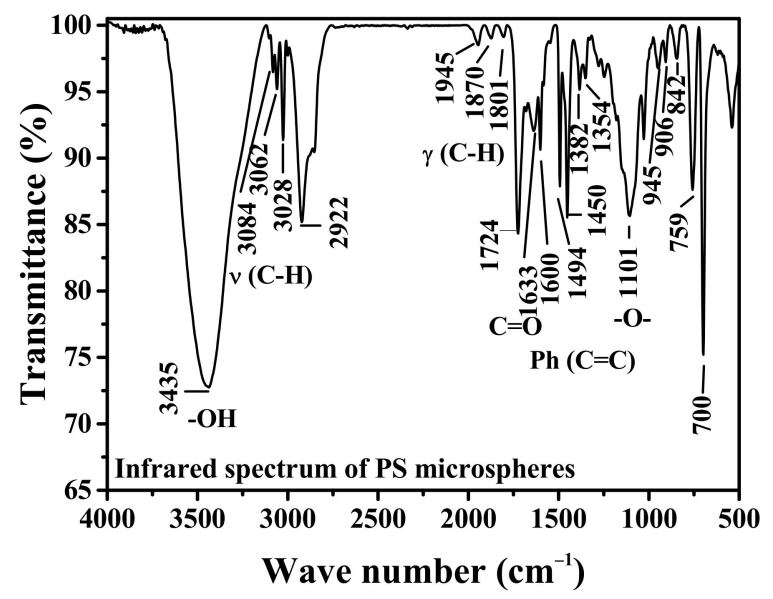

Figure 5. Infrared spectrum of PS microspheres.

The morphology of PS microspheres was observed by SEM and TEM. Figure 6a shows the SEM image of PS microspheres with the same scale. Clearly, PS microspheres have a smooth surface, compact arrangement, and a diameter of about $100 \mathrm{~nm}$. PS microspheres are visualized by transmission electron microscopy. As shown in Figure $6 \mathrm{~b}$, it can be seen that that the core-shell PS microspheres have an average diameter of about $100 \mathrm{~nm}$. The shell looks very uniform with a thickness of about $11 \mathrm{~nm}$. The PS microspheres with styrene as core, hydroxyethyl methacrylate and polyethylene glycol methyl ether methacrylate as shell were successfully prepared by soap-free emulsion polymerization.

(a)

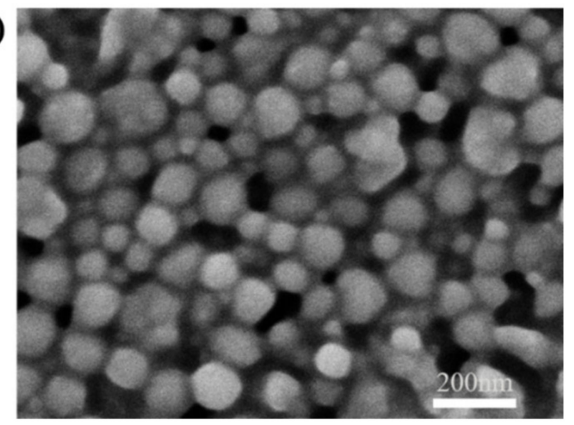

(b)

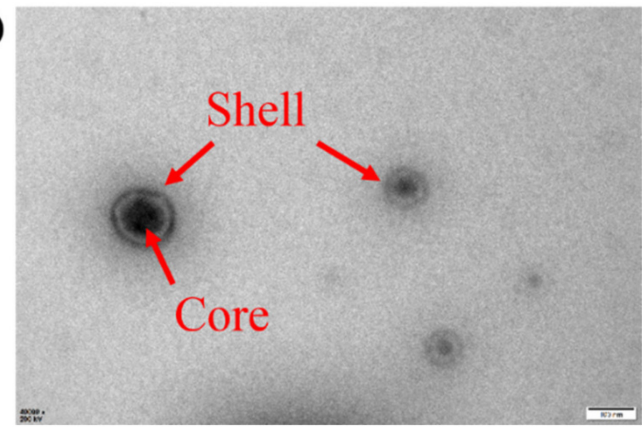

Figure 6. Microstructure of PS microspheres (a) SEM image; (b) TEM image.

\subsection{Chemical Structure and Morphology of PS-PEG Hydrogels}

ATR-FTIR tests were carried out on the PS-NCO intermediate terminated with isocyanate through bulk polymerization and the PS-PEG hydrogel as the final product. The infrared spectra obtained were showed in Figures $7 \mathrm{a}$ and $7 \mathrm{~b}$, respectively. There is a very obvious absorption peak at $2258 \mathrm{~cm}^{-1}$ in Figure $7 \mathrm{a}$. This is the absorption peak of isocyanate (-NCO), which proves that the synthetic PS-NCO intermediate will successfully seal the isocyanate. As showed in Figure 7b, PS-PEG hydrogels with different contents were successfully synthesized. It can be observed from the infrared spectrum that the stretching vibration absorption peak of the -NH bond is located at $3396 \mathrm{~cm}^{-1}$, and its deformation vibration peak is at $1546 \mathrm{~cm}^{-1}$. In addition, at $1643 \mathrm{~cm}^{-1}$ and $1701 \mathrm{~cm}^{-1}$, the stretching vibration peak of $\mathrm{C}=\mathrm{O}$ in the amide carbonyl group and the stretching vibration peak of $\mathrm{C}=\mathrm{O}$ in the urea group is separated. The above characteristic peaks indicate that the final product is PEG-based hydrogel. The skeleton vibration absorption peak of the benzene ring is at $1450 \mathrm{~cm}^{-1}, \mathrm{C}-\mathrm{O}$ stretching vibration peak is at $1248 \mathrm{~cm}^{-1}$, and the strong absorption peak at $1097 \mathrm{~cm}^{-1}$, is the -O- asymmetric stretching vibration peak polyether structure, which indicates that PEG-based hydrogel successfully introduces the structure containing benzene ring and polyether. Moreover, there was no obvious absorption peak between $2200 \mathrm{~cm}^{-1}$ and $2300 \mathrm{~cm}^{-1}$, indicating that the isocyanate in the polymer had been 
thoroughly reacted at this time, and the final synthetic substance was PS-PEG composite hydrogel.

(a)

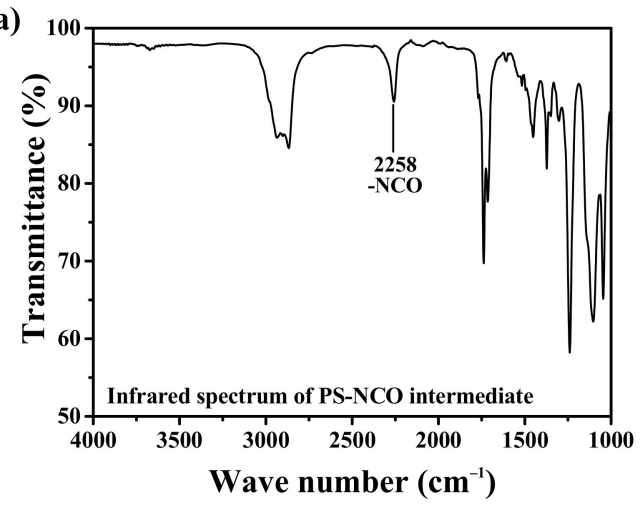

(b)

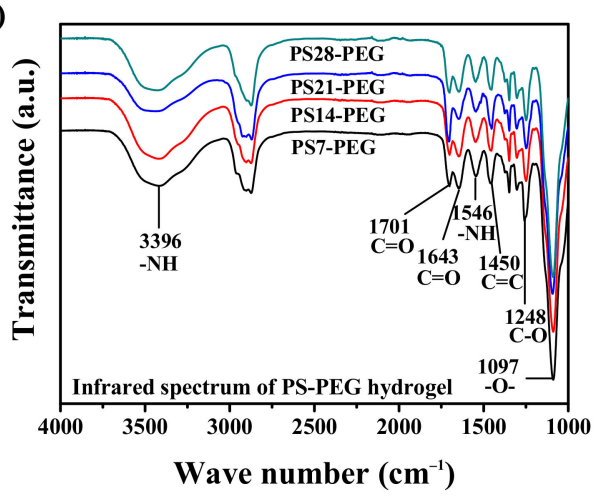

(c)

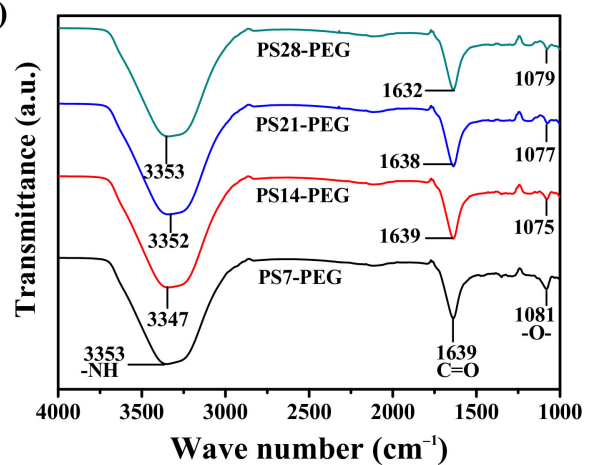

Figure 7. FTIR spectra of studied samples (a) PS-NCO intermediates; (b) PS-PEG hydrogel; (c) PS-PEG hydrogel swelling in deionized water for $48 \mathrm{~h}$.

The hydrogel sample after soaking and swelling in deionized water for $48 \mathrm{~h}$, as showed in Figure 7c. The absorption peak of free - NH formed hydrogen bond with carbamate was located at $3340 \mathrm{~cm}^{-1}$. In contrast, the carbonyl peak $\mathrm{C}=\mathrm{O}$ after soaking in different solutions was redshifted to about $1638 \mathrm{~cm}^{-1}$, and the ether bond region was also redshifted from $1097 \mathrm{~cm}^{-1}$ to $1074 \mathrm{~cm}^{-1}$. The main reason is that a large number of water molecules in various solutions provide the $-\mathrm{H}$ receptor, which increases the degree of hydrogen bonding.

The above analysis showed that the PS-PEG hydrogel was successfully synthesized. After wet curing, the free - $\mathrm{NH}$ and -CO- stretching vibration region were split into two peaks, namely, the free absorption peak and the absorption peak forming hydrogen bond. Meanwhile, the isocyanate was consumed, and after soaking, the - $\mathrm{NH}$ and - $\mathrm{CO}$ - on the hydrogel structure tended to form a hydrogen bond with water molecules. As a result, the number of hydrogen bonds increases, but the number of hydrogen bonds on the segment structure decreases, which weakens the degree of hydrogen bonding of the hydrogel structure chain.

SEM observation showed PS microspheres were uniformly dispersed in PS-PEG hydrogels, refers in Figure 8a. XRD spectra of PS microspheres and PS14-PEG hydrogel were shown in Figure $8 \mathrm{~b}$. Clearly, there was a broad peak near $2 \theta$ of $19.9^{\circ}$, which was a characteristic amorphous diffraction peak, and it was consistent with the literature [32]. There were two dominant characteristic peaks respectively at $19.4^{\circ}$ and $23.5^{\circ}$ which derived from the PEG, which were consistent with the literature [33]. However, it can be observed that the amorphous peak of PS shifted about $0.9^{\circ}$ in the hydrogel by compared with two diffraction peaks, which due to the distortion of the crystal lattice caused by the internal residual stress of hydrogel. In this regard, DSC is further used to perform differential thermal analysis on each hydrogel sample, it can be clearly observed that the PS-PEG 
hydrogels added with PS microspheres all have an exothermic crystallization peak at about $-33{ }^{\circ} \mathrm{C}$ in Figure 8c. In summary, combined with the infrared spectrum, the PS microspheres were successfully compounded with the PEG-based hydrogel.
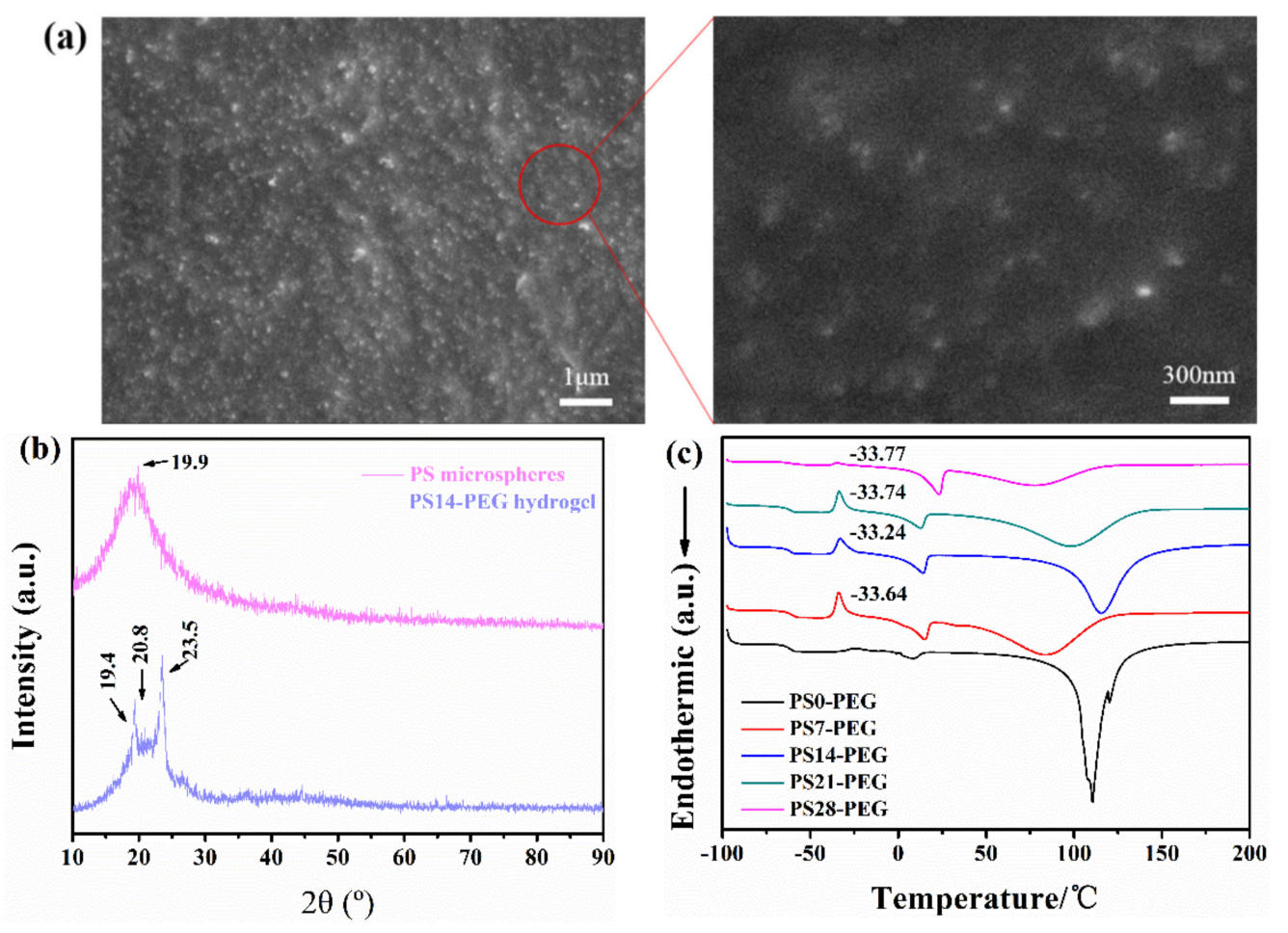

Figure 8. (a) SEM image of PS-PEG hydrogel; (b) XRD spectra of PS14-PEG hydrogel; (c) DSC curves of PS-PEG hydrogel.

The morphology diagram of PS-PEG composite hydrogel with each PS microspheres addition number was clearly as showed in Figure 9. The observation results also indicated that with the increase of PS microspheres, the light transmittance of the composite hydrogel decreased gradually. While the macroscopic light transmittance of the PS0-PEG sample without PS microspheres is the strongest.

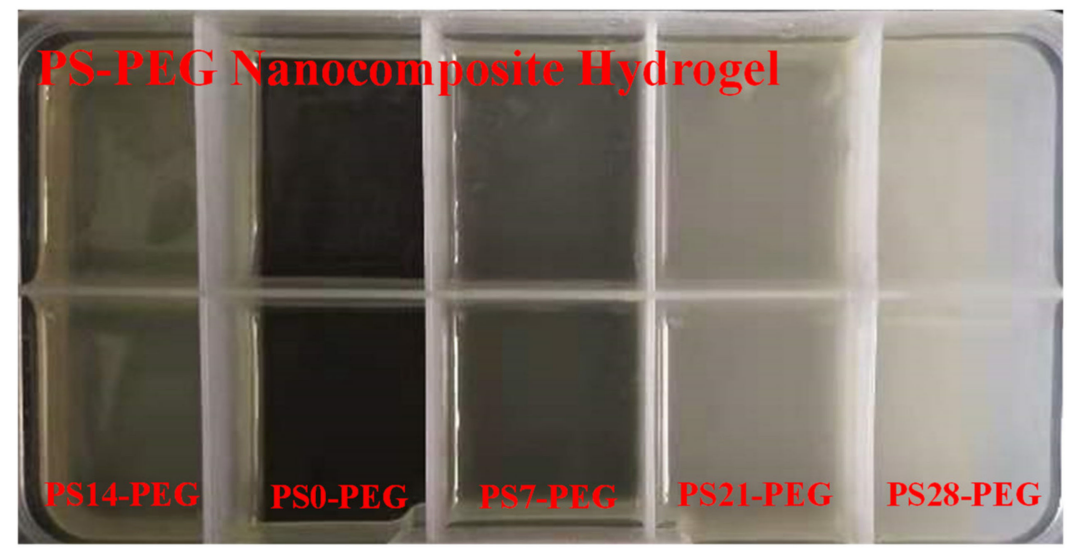

Figure 9. Morphology of PS-PEG hydrogel.

\subsection{Swelling Properties of Hydrogels}

The swelling properties of each sample of PS-PEG hydrogel placed in deionized water for $48 \mathrm{~h}$ were showed in Figure 10a. In the first $3 \mathrm{~h}$ of the initial swelling stage, the swelling degree of each sample increased significantly in water absorption. With the increase contents of PS microspheres, PS-PEG hydrogels had rapid water absorption and swelling 
property in the first $6 \mathrm{~h}$. Then the water absorption and swelling degree slowed down, but it still kept increasing. As the PS microspheres continued to increase, the swelling degree of the PS28-PEG sample was smaller than that of the PS21-PEG sample; that is, the swelling degree increased first and then decreased. This is because the swelling of hydrogel is determined by hydrogen bonding and hydrophobicity, and the PS microspheres contain oleophilic groups simultaneously. When the content exceeds a certain proportion, the water absorption of the hydrogen bond is less than that of its hydrophobic structure so that the swelling degree will be affected to a certain extent. At the same time, we calculated the swelling degree of each hydrogel sample after soaking for $24 \mathrm{~h}$ and $48 \mathrm{~h}$, as listed in Table 1. It demonstrates that the PS21-PEG sample had the highest swelling degree, not the PS28-PEG, which is because the dense three-dimensional cross-linking structure is formed after the third step of polymerization. However, the limited space of the system will further hinder the absorption of water by the hydrogel.

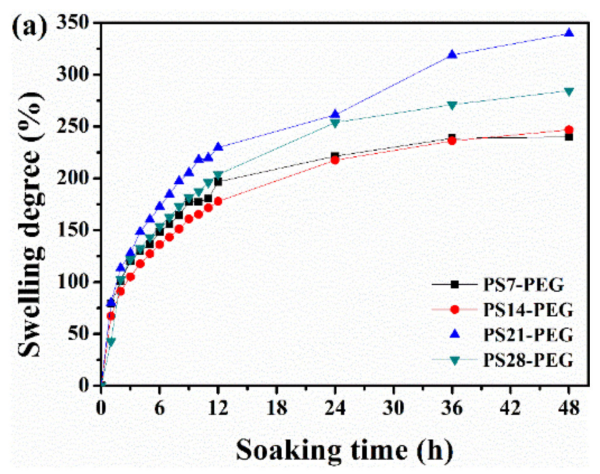

(b)

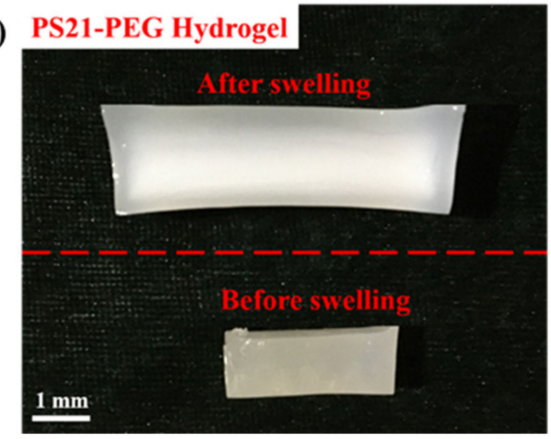

Figure 10. (a) Swelling degree with soaking time; (b) morphology of PS21-PEG hydrogel before and after swelling.

Table 1. Swelling degree of studied samples.

\begin{tabular}{ccccc}
\hline Sample & PS7-PEG & PS14-PEG & PS21-PEG & PS28-PEG \\
\hline $\begin{array}{c}\text { Swelling degree } \\
\text { at } 24 \mathrm{~h}(\%)\end{array}$ & 221.51 & 217.70 & 261.34 & 253.85 \\
\hline $\begin{array}{c}\text { Swelling degree } \\
\text { at } 48 \mathrm{~h}(\%)\end{array}$ & 239.78 & 246.90 & 339.64 & 284.62 \\
\hline
\end{tabular}

The volume of PS21-PEG hydrogel after swelling for $48 \mathrm{~h}$ increases to about 3.4 times that of original dry gel sample, as shown in Figure 10b. It can be observed from the figure that the volume of the hydrogel has undergone macroscopic expansion after swelling. It always keeps a certain swelling degree in deionized water. This is because the - $\mathrm{OH}$ of the PS shell layer with a core-shell structure can be cross-linked with -NCO, making the PS microspheres a 'central connection point', which promotes the overall hydrogel the three-dimensional structure was significantly improved.

\subsection{Contact Angle and Surface Energy}

Since static contact angle is measured under equilibrium condition, it can only reflect the wettability at equilibrium, and cannot reveal the change information of surface structure. It is unable to study the relationship between material surface structure and wettability, as well as the precise control of the surface structure. However, dynamic contact angle just makes up this shortcoming. It can provide information on surface roughness, uniformity of chemical properties, reconstruction of hydrophilic/hydrophobic chain segments. The dynamic water contact angle (DWCA) and dynamic diiodomethane contact angle (DDCA) of PS-PEG are calculated for $5 \mathrm{~min}$, respectively. The surface free energy is calculated as listed in Table 2. 
Table 2. WCA and DCA and surface free energy of PS- hydrogel samples.

\begin{tabular}{cccc}
\hline Sample & WCA $\left({ }^{\circ}\right)$ & DCA $\left({ }^{\circ}\right)$ & $\begin{array}{c}\text { Surface Free Energy } \\
\left(\mathbf{m J} / \mathbf{m}^{\mathbf{2}}\right)\end{array}$ \\
\hline PS7-PEG & $107 \pm 0.33$ & $50.5 \pm 0.41$ & $35.52 \pm 0.19$ \\
PS14-PEG & $104 \pm 0.24$ & $49.25 \pm 0.33$ & $35.69 \pm 0.16$ \\
PS21-PEG & $102.5 \pm 0.36$ & $55.5 \pm 0.28$ & $31.49 \pm 0.13$ \\
PS28-PEG & $97 \pm 0.22$ & $55 \pm 0.42$ & $31.45 \pm 0.24$ \\
\hline
\end{tabular}

PS-PEG hydrogel was tested in deionized water for DWCA, as showed in Figure 11a. The initial DWCA was PS7-PEG > PS14-PEG > PS21-PEG > PS28-PEG. The final state DWCA was PS21-PEG > PS14-PEG > PS7-PEG > PS28-PEG. As the number of PS microspheres increased, the initial DWCA became lower and changed from hydrophobic to hydrophilic within $5 \mathrm{~min}$ (from $104^{\circ}$ to $75.5^{\circ}$ in the case of PS14-PEG). This was because the PS surface of the PS-PEG hydrogel compound with PS microspheres powder was rich in a large $-\mathrm{OH}$, which was a strong hydrophilic group. In the subsequent synthesis process, after the reaction with isocyanate and wet solidification of the film, the chain segment formed by it is more flexible. Therefore, for the test area of water droplets, the more flexible hydrophilic chain segment is more likely to migrate and enrich on the surface of the coating, realizing the conversion process from hydrophobic to hydrophilic, and then forming a layer of hydration film. Therefore, it appears to be strongly hydrophilic after the appearance of hydrophobicity. It also can be observed from the swelling test.
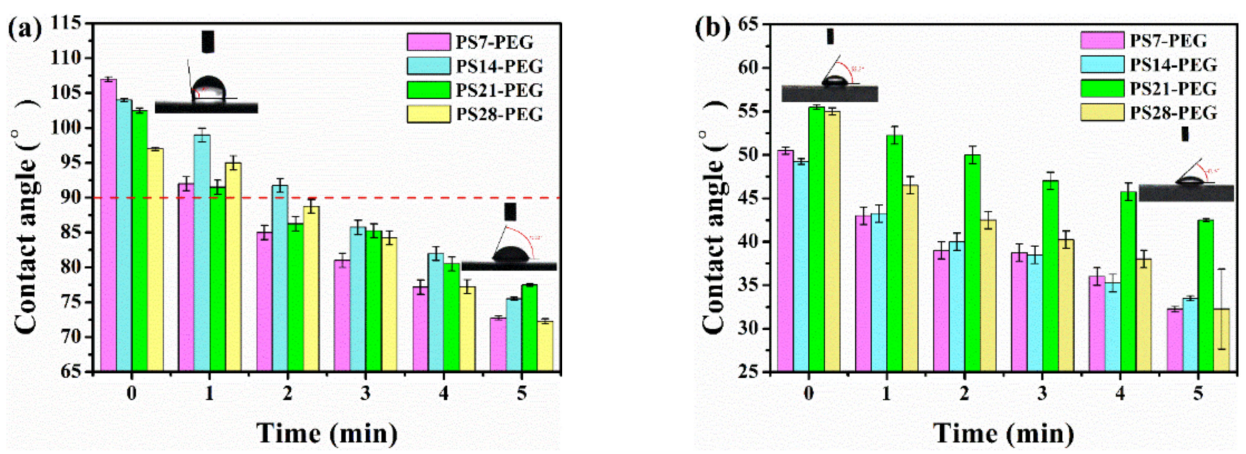

Figure 11. PS-PEG hydrogel dynamic contact angle (a) DWCA; (b) DDCA.

The results after the DDCA test are plotted as showed in Figure 11b. It is not difficult to observe that the initial DDCA is PS21-PEG $>$ PS28-PEG $>$ PS7-PEG $>$ PS14-PEG. The final DDCA is PS21-PEG > PS14-PEG > PS7-PEG > PS28-PEG. All samples are lipophilic (take PS14-PEG as an example, from $49.25^{\circ}$ to $33.5^{\circ}$ ). In addition, DWCA and DDCA showed the same change trend. PS-PEG hydrogels has both hydrophilic and lipophilic, when the surface contacts with polar or non-polar media, internal structure of the hydrogel will be restructured within a certain period of time, and the corresponding functional groups will eventually accumulate on the surface through the turnover of the flexible chain.

The surface energy of the coating was calculated according to the given formula by measuring the water contact angle and diiodomethane contact angle. The atoms (or ions) on the surface are in a state of imbalance between internal and external forces. The more unbalanced the force is, the more unstable it is and the higher its surface energy is. Therefore, as evidenced by the surface energy of hydrogel can be reduced by adding PS microspheres properly, and the surface energy decreased with the increase of PS microspheres. The surface of PS28-PEG sample is minimum, only $31.45 \mathrm{~mJ} / \mathrm{m}^{2}$.

\subsection{Surface Morphology and Roughness}

The relationship between the surface roughness of the coating before and after soaking and the content of PS microspheres was showed in Figure 12. The surface roughness of the 
hydrogel before immersion increased with increasing the content. This is because the nanoPS microspheres have high surface activity. Therefore, too many nanospheres will increase the agglomeration effect. The agglomeration effect is enhanced, small agglomerations are easily formed in the partially incompletely dispersed areas, which increases the surface roughness.

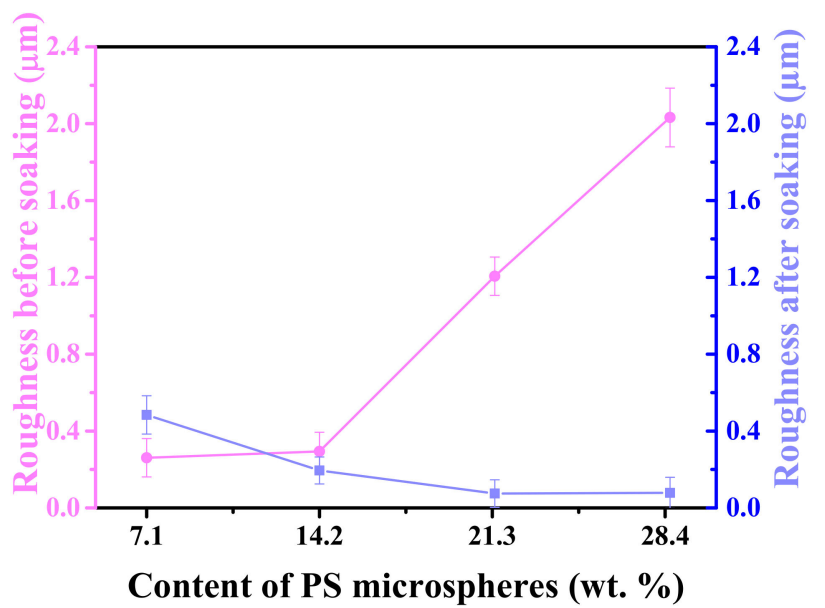

Figure 12. Roughness of PS-PEG hydrogels before and after swelling for $48 \mathrm{~h}$.

When the sample swelled for $48 \mathrm{~h}$, the surface roughness of the sample decreased with the increase of PS microspheres. This is because PS microspheres are used as the crosslinking points in the hydrogel, making the crosslinking density increase, it caused three-dimensional space to become more compact when the hydrogel absorbs water, it can form a hydration layer on the surface, and the roughness of the surface is reduced. Therefore, the surface of the hydrogel after swelling at the same time is more even and smooth. As for PS14-PEG hydrogel with PS microspheres added number of $14.2 \mathrm{wt} \%$, the roughness of PS14-PEG hydrogel was not only low before soaking, but also decreased significantly after soaking for $48 \mathrm{~h}$, which was better than that of other samples.

\subsection{Compression Performance}

The rheological test cannot reflect the macroscopic fracture property of the material under large deformation, so it is necessary to test the compression property of the hydrogel.

The results showed that a simple compression test of PS-PEG hydrogel, as showed in Figure 13. It was observed that the hydrogel can recover to its original shape after the removal of external force without being damaged, with certain elasticity.

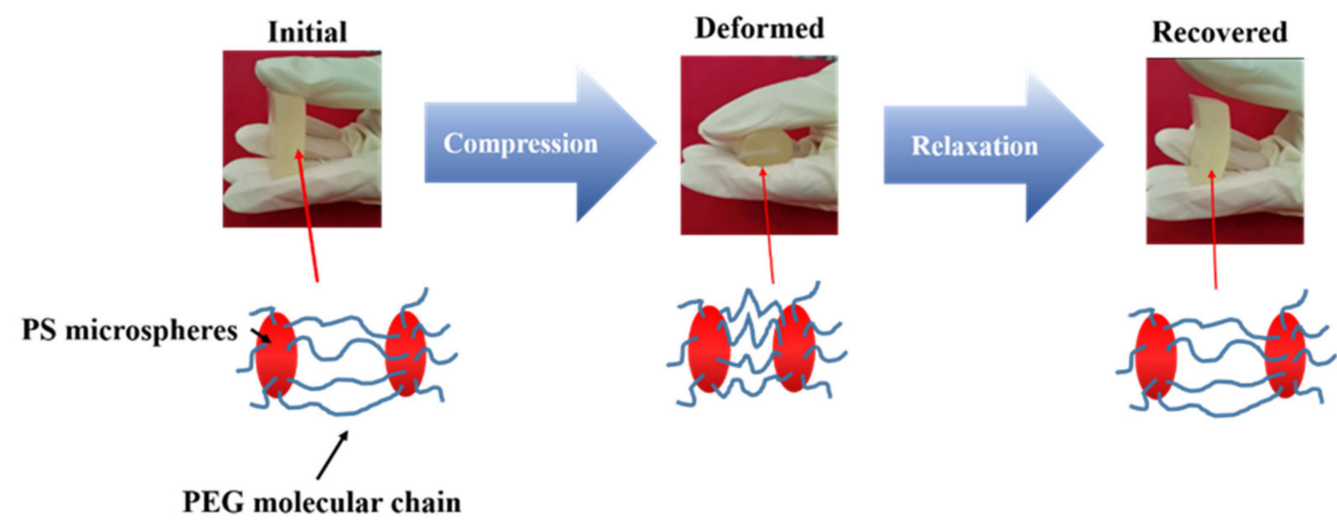

Figure 13. Schematic diagram of compression deformation and recovery of PS-PEG hydrogel. 
The compressive stress-strain curves, compressive elastic modulus and maximum compressive stress of all samples were showed in Figure 14a,b. Obviously, the maximum compression stress of PS14-PEG hydrogel reached 5.83 MPa, which was nearly 3.3 times higher than that of PS7-PEG hydrogel $1.78 \mathrm{MPa}$. However, the maximum compression stress gradually decreased with increasing PS microspheres, and the maximum compression stress of PS28-PEG hydrogel was $2.89 \mathrm{MPa}$. The maximum compression stress of PS28-PEG is 2 times lower than that of PS14-PEG. The maximum compressive stress of PS14-PEG hydrogel is much higher than that reported by Jiang [34]. He used hyperbranched polyethyleneimine (HPEI) as the main material and prepared the quaternary ammonium nanoparticles, these nanoparticles were added to HPEI to prepare hydrogels. The maximum compressive stress of his hydrogels reached nearly $2.5 \mathrm{MPa}$.
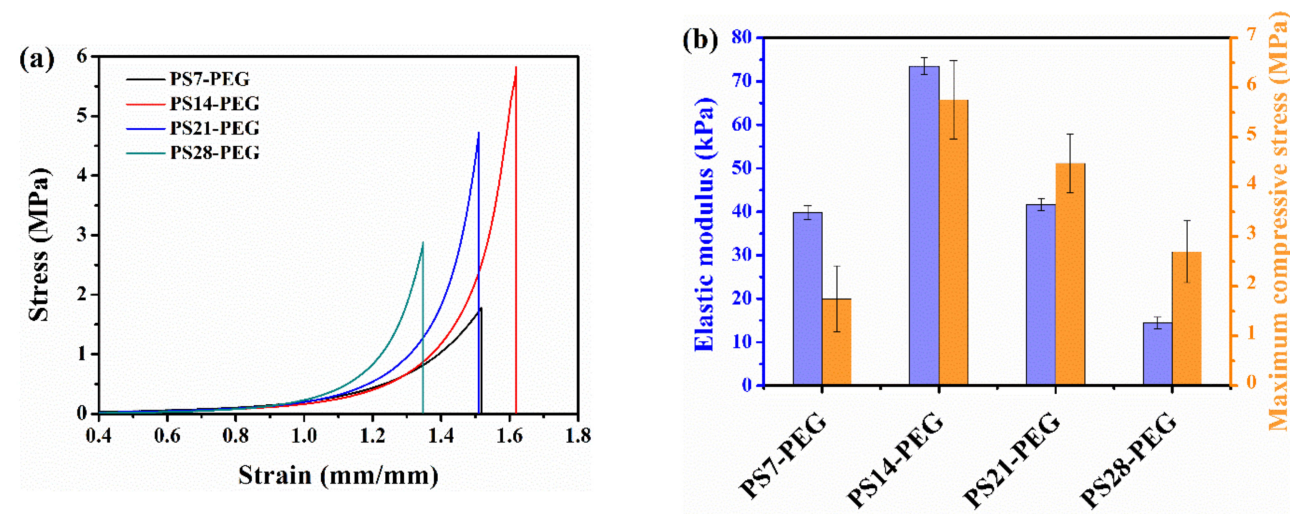

Figure 14. Mechanical properties of PS-PEG hydrogels (a) compressive stress-strain curves; (b) elastic modulus and maximum compressive stress.

Similarly, the relative compression ratio increased first and then decreased with the increase of PS microspheres. The relative compression ratio of PS14-PEG hydrogel reached 161.96\%, while PS21-PEG and PS28-PEG were damaged at $151.04 \%$ and $134.71 \%$, respectively. This indicates that the proper content of PS microspheres can effectively improve the compression performance of the composite hydrogels. However, the excessive PS microspheres will increase the density of hydrogen bonds and crosslinking points between the segments in the hydrogel. The increasing density of overall three-dimensional network structure makes chain segments difficult to move.

The compressive elastic modulus was fitted by the stress-strain curve. With increasing the content of PS microspheres from $7.1 \mathrm{wt} \%$ to $14.2 \mathrm{wt} \%$, the maximum compression modulus increased from $39.6 \mathrm{kPa}$ to $73.7 \mathrm{kPa}$, the latter was 1.86 times higher than the former. Afterwards, the compressive modulus of the hydrogel decreased gradually, and the PS28-PEG hydrogel was only $14.8 \mathrm{kPa}$. Compared with PS14-PEG, the reduction was nearly five-fold. The results showed that too many PS microspheres can easily lead to their agglomeration, this leads to uneven distribution of internal structures. Too many rigid PS microspheres reduced the elastic modulus and compressive strength of hydrogels.

The dispersion of low-concentration nanoparticles was relatively uniform. The molecular chain between two nanoparticles was longer, so the degree of molecular chain curl was higher. A large number of energy were consumed in the compression process. Therefore, the PS-PEG hydrogels exhibit some elasticity at low concentrations. With the increase of PS microspheres content, the cross-linking density of hydrogel increased, it resulted in a shorter chain between the two nanoparticles, so the elasticity goes down. At this time, when the hydrogel is extruded by compression, the space size of the microspheres were irreversibly destroyed to a certain extent.

\section{Discussion}

The above results demonstrate that the content of PS microspheres not only affects the surface properties of composite hydrogels, but also affects the physical properties 
and mechanical properties of composite hydrogels. With the increase of the content of PS microspheres, the mechanical properties of the hydrogel gradually increase, as showed in Figure 15a. when the content of PS microspheres reaches $14.2 \mathrm{wt} \%$, the mechanical properties of the hydrogel are the maximum, while the corresponding the swelling degree was lower at $48 \mathrm{~h}$, indicating that the internal cross-linking structure of the hydrogel is the tightest; while continuing to increase the number of PS microspheres added, the mechanical properties show a downward trend. In addition, the surface roughness and surface energy of the hydrogel decreased with the increase of PS microspheres, as showed in Figure 15b.
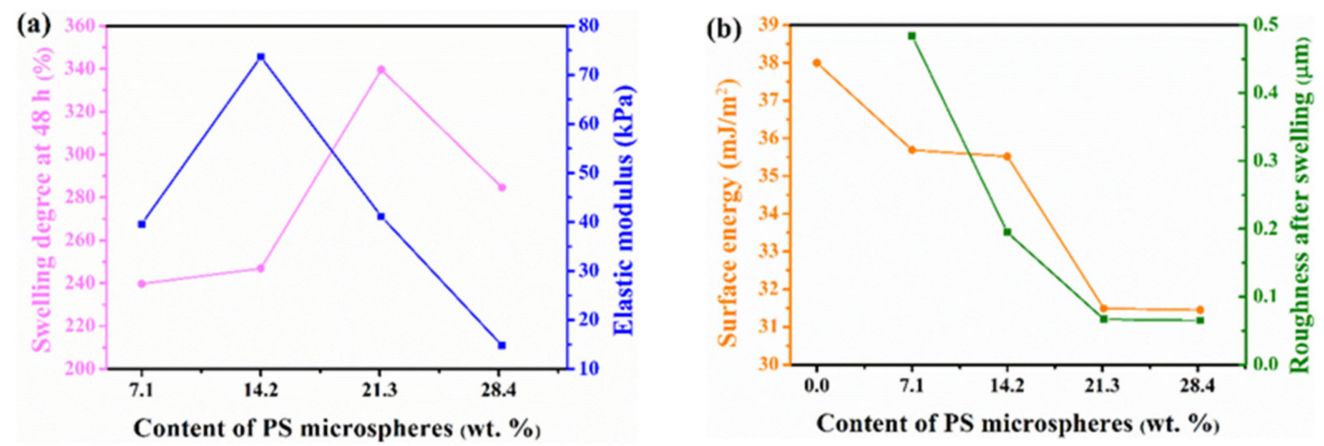

Figure 15. Changes in various properties with the content of PS microspheres (a) swelling degree and mechanical properties; (b) surface energy and roughness.

The deep-frozen fracture morphology of hydrogel samples was shown in the Figure 16. The observation indicates that the content of PS microspheres obviously affect the fracture morphology of the hydrogel. The fracture of PS7-PEG hydrogel has a certain arrangement of gully shape, as showed in Figure 16a. This is because a few PS microspheres can make the three-dimensional network crosslinking structure of the hydrogel more compact. However, the number of connection points is limited and the crosslinking structure is not perfect, so the segment with weak bonding strength breaks when subjected to external stress. When the number of microspheres continued to increase, the fracture groove of PS14-PEG hydrogel was significantly less than that of PS7-PEG hydrogel, as showed in Figure $16 \mathrm{~b}$, and has a certain regularity, is not along the only direction of fracture. This is because the hydrogel will deform under the action of natural forces, in the place where the spatial structure of hydrogel is cross-linked closely, the bond strength is high and it is not easy to be broken, while in the place where the relative bond strength is low, it is easy to reach the limit and break. The fracture occurs in the position where the relative strength of the hydrogel bonding is poor, so the whole fracture process will be affected by the distribution characteristics of this region. Therefore, the fracture position is different at this time, and it has a certain spatial structure regularity.

(a)

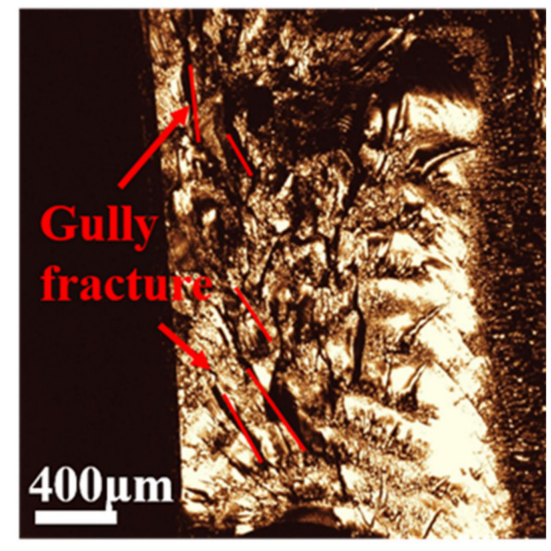

(b)

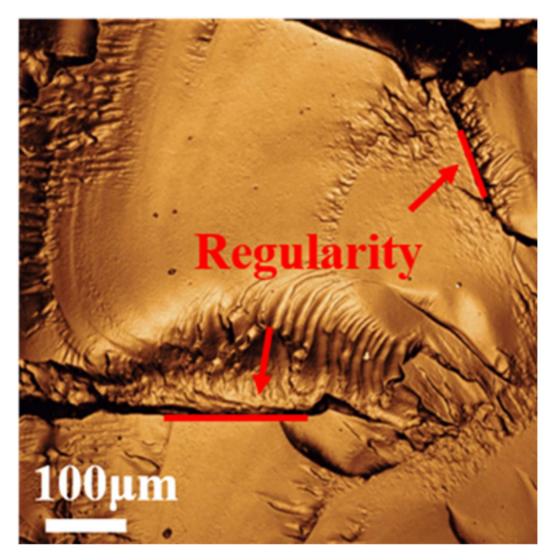

Figure 16. Cont. 
(c)

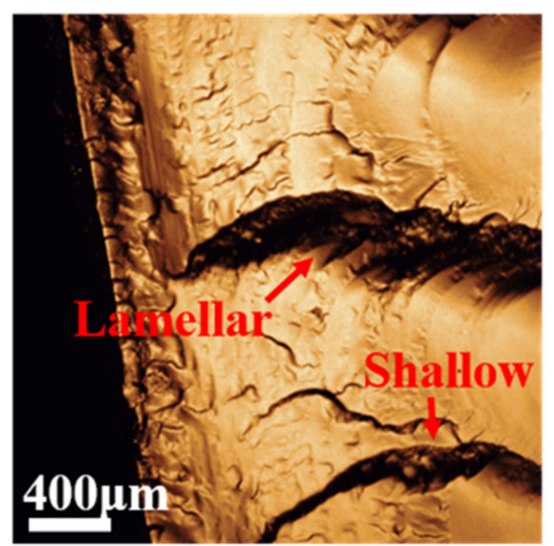

(d)

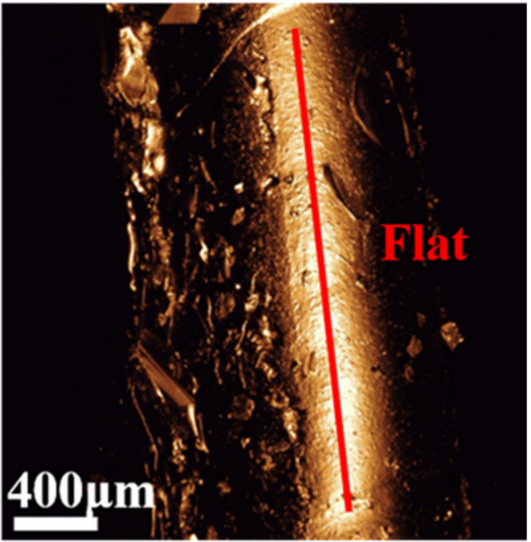

Figure 16. Fracture morphology of PS-PEG hydrogels. (a) PS7-PEG. (b) PS14-PEG. (c) PS21-PEG. (d) PS28-PEG.

Continue to increase PS microspheres, as showed in Figure 16c, the PS21-PEG hydrogel cross section is similar to lamellar fault, and the depth of the gully produced by the fracture cracks is relatively shallow. The reason is that the internal crosslinking structure continues to increase and the binding strength increases with the further increase of the content number of nano-spheres. Therefore, in the whole process of fracture, the strength of longitudinal fracture cannot extend well to the interior, it only keeps the regular fracture in the transverse direction. The corresponding PS28-PEG hydrogel had the most PS microspheres, as showed in Figure 16d. The results demonstrates that its fracture morphology was relatively flat, and there was no obvious fracture layer and similar gully shape. This was attributed to the fact that the sample contains the most PS microspheres, and the full cross-linking reaction inside the hydrogel results in the highest binding strength, thus forming a three-dimensional structure with high strength, which delays the further fault damage of the internal structure caused by the external force. Consequently, the influence of PS microspheres content on fracture strength can be generalized schematically as showed in Figure 17.

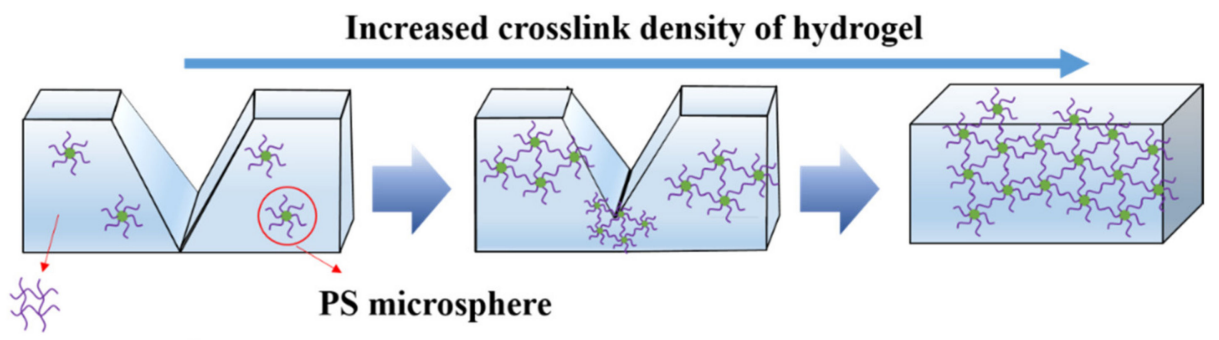

PEG hydrogel matrix

Figure 17. Influence of PS microspheres content on fracture strength.

\section{Conclusions}

(1) PS microspheres with a core-shell structure were successfully prepared by soap-free emulsion polymerization. The diameter of the microspheres was about $100 \mathrm{~nm}$ and the shell thickness was about $11 \mathrm{~nm}$. Moreover, PS microspheres reinforced PEGbased hydrogels were successfully prepared by bulk polymerization and solution polymerization.

(2) The swelling degree of PS-PEG hydrogel changes with the content of PS microsphere. When its content was $14.2 \mathrm{wt} \%$, its swelling degree was $246.9 \%$ at $48 \mathrm{~h}$. PS-PEG hydrogels have good swelling performance.

(3) The static water contact angle and surface free energy of the hydrogels decreased with increasing PS microspheres content, but diiodomethane contact angle creased. The surface energy of PS28-PEG hydrogel is only $31.45 \mathrm{~mJ} / \mathrm{m}^{2}$. Moreover, the surface of 
PS-PEG hydrogel can changed from hydrophobic to hydrophilic during the wetting process.

(4) The content of PS microspheres can affect the surface roughness of the hydrogel. The surface roughness of the dry gel increases with the increase content of microspheres. However, the surface roughness of the soaked PS-PEG hydrogel decreased with the content of PS microspheres.

(5) Appropriate content of PS microspheres can improve the mechanical properties of PS-PEG hydrogels. When the content of PS microspheres is $14.2 \mathrm{wt} \%$, the maximum compressive stress reaches $5.83 \mathrm{MPa}$, the relative compression ratio reaches $161.96 \%$, and the compressive elastic modulus is $73.7 \mathrm{kPa}$.

Author Contributions: C.Z., Z.Z. and Y.Q., conceptualization; C.Z., Z.Z. and Y.Q., methodology; C.Z., software; C.Z., validation; C.Z. and Z.Z., formal analysis; C.Z. and Z.Z., investigation; Y.Q. and Z.Z., resources; C.Z., data curation; C.Z., writing-original draft preparation; C.Z. and Z.Z., writing-review and editing; C.Z., visualization; Z.Z. and Y.Q., supervision; Z.Z. and Y.Q., project administration; Z.Z. and Y.Q., funding acquisition. All authors have read and agreed to the published version of the manuscript.

Funding: This research was funded by the Project of Equipment Pre-research Field Fund, grant number 61409220304 and the National Natural Science Foundation of China, grant number 51879021.

Institutional Review Board Statement: Not applicable.

Informed Consent Statement: Not applicable.

Data Availability Statement: The data in this study are contained within the article.

Conflicts of Interest: The authors declare no conflict of interest.

\section{References}

1. Naahidi, S.; Jafari, M.; Logan, M.; Wang, Y.; Yuan, Y.; Bae, H.; Dixon, B.; Chen, P. Biocompatibility of hydrogel-based scaffolds for tissue engineering applications. Biotechnol. Adv. 2017, 35, 530-544. [CrossRef]

2. Bittner, S.M.; Guo, J.L.; Melchiorri, A.; Mikos, A.G. Three-dimensional printing of multilayered tissue engineering scaffolds. Mater. Today 2018, 21, 861-874. [CrossRef] [PubMed]

3. Ahmed, E.M. Hydrogel: Preparation, characterization, and applications: A review. J. Adv. Res. 2015, 6, 105-121. [CrossRef] [PubMed]

4. Wichterle, O.; Lim, D. Hydrophilic gels for biological use. Nature 1960, 185, 117-118. [CrossRef]

5. Zhang, Y.S.; Khademhosseini, A. Advances in engineering hydrogels. Science 2017, 356, 80-81. [CrossRef]

6. Li, L.; Wang, Y.; Pan, L.; Shi, Y.; Cheng, W.; Shi, Y.; Yu, G. A nanostructured conductive hydrogels-based biosensor platform for human metabolite detection. Nano Lett. 2015, 15, 1146-1151. [CrossRef] [PubMed]

7. Grehan, L.; Killion, J.; Devery, S.; Higginbotham, C.; Geever, L. Biological evaluation and characterisation of novel hydrogel matrices as scaffolds for bone tissue engineering. Appl. Mech. Mater. 2014, 679, 63-75. [CrossRef]

8. Kamata, H.; Akagi, Y.; Kayasuga-Kariya, Y.; Chung, U.I.; Sakai, T. “Nonswellable” hydrogel without mechanical hysteresis. Science 2014, 343, 873-875. [CrossRef] [PubMed]

9. Hu, X.; Tan, H.; Chen, P.; Wang, X.; Pang, J. Polymer micelles laden hydrogel contact lenses for ophthalmic drug delivery. J. Nanosci. Nanotechnol. 2016, 16, 5480-5488. [CrossRef]

10. Sato, K.; Nakajima, T.; Hisamatsu, T.; Nonoyama, T.; Kurokawa, T.; Gong, J.P. Phase-separation-induced anomalous stiffening, toughening, and self-healing of polyacrylamide gels. Adv. Mater. 2015, 27, 6990-6998. [CrossRef]

11. Yuan, J.; Wen, D.; Gaponik, N.; Eychmüller, A. Enzyme-encapsulating quantum dot hydrogels and xerogels as biosensors: Multifunctional platforms for both biocatalysis and fluorescent probing. Angew. Chem. Int. Ed. 2013, 52, 976-979. [CrossRef]

12. Liu, S.; Guo, W. Anti-biofouling and healable materials: Preparation, mechanisms, and biomedical applications. Adv. Funct. Mater. 2018, 28, 1-25. [CrossRef]

13. Yao, J.; Chen, S.; Ma, C.; Zhang, G. Marine anti-biofouling system with poly( $\varepsilon$-caprolactone)/clay composite as carrier of organic antifoulant. J. Mater. Chem. B 2014, 2, 5100-5106. [CrossRef]

14. Qiu, Y.; Park, K. Environment-sensitive hydrogels for drug delivery. Adv. Drug Deliv. Rev. 2012, 64, 49-60. [CrossRef]

15. Eswaramma, S.; Rao, K.S.V.K. Synthesis of dual responsive carbohydrate polymer based IPN microbeads for controlled release of anti-HIV drug. Carbohydr. Polym. 2017, 156, 125-134. [CrossRef]

16. Li, L.; Li, H.S.; Pauza, C.D.; Bukrinsky, M.; Zhao, R.Y. Roles of HIV-1 auxiliary proteins in viral pathogenesis and host-pathogen interactions. Cell Res. 2005, 15, 923-934. [CrossRef] 
17. Liu, R.; Liang, S.; Tang, X.Z.; Yan, D.; Li, X.; Yu, Z.Z. Tough and highly stretchable graphene oxide/polyacrylamide nanocomposite hydrogels. J. Mater. Chem. 2012, 22, 14160-14167. [CrossRef]

18. Wu, Y.; Zhou, Z.; Fan, Q.; Chen, L.; Zhu, M. Facile in-situ fabrication of novel organic nanoparticle hydrogels with excellent mechanical properties. J. Mater. Chem. 2009, 19, 7340-7346. [CrossRef]

19. Wu, Y.; Xia, M.; Fan, Q.; Zhu, M. Designable synthesis of nanocomposite hydrogels with excellent mechanical properties based on chemical cross-linked interactions. Chem. Commun. 2010, 46, 7790-7792. [CrossRef]

20. Shao, Q.L.; Liao, Q.; Wang, H.Y. Synthesis and characterization of smart core-shell nanogels. J. Funct. Polym. 2012, 25, 47-51. [CrossRef]

21. Core, P.; Microgels, S.; Behavior, S.; Hellweg, T.; Dewhurst, C.D.; Eimer, W.; Kratz, K.; Microgels, P.C.S. PNIPAM-co-polystyrene core-shell microgels: Structure, swelling behavior, and crystallization. Langmuir 2004, 20, 4330-4335. [CrossRef]

22. Berndt, I.; Richtering, W.; Kiel, D. Doubly temperature sensitive (core-shell) microgels. Macromolecules 2003, 36, 8780-8785. [CrossRef]

23. Jones, C.D.; Lyon, L.A. Dependence of shell thickness on core compression in acrylic acid modified poly(N-isopropylacrylamide) core/shell microgels. Langmuir 2003, 19, 4544-4547. [CrossRef]

24. Makino, K.; Yamamotoa, S.; Fujimoto, K.; Kawaguchi, H.; Ohshima, H. Surface structure of latex coverd with temperatur-sensitive hydrogel layers. J. Colloid Interface Sci. 1994, 166, 251-258. [CrossRef]

25. Pelton, R.H.; Chibante, P. Preparation of aqueous latices with N-isopropylacrylamide. Colloids Surfaces 1986, $20,247-256$. [CrossRef]

26. Juodkazis, S.; Mukai, N.; Wakaki, R.; Yamaguchi, A.; Matsuo, S.; Misawa, H. Reversible phase transitions in polymer gels induced by radiation forces. Nature 2000, 408, 178-181. [CrossRef]

27. Tang, S.; Hu, Z.; Cheng, Z.; Jianzhong, W. Crystallization kinetics of thermosensitive colloids probed by transmission spectroscopy. Langmuir 2004, 20, 8858-8864. [CrossRef]

28. Zheng, X.; Hao, Y.; Zhang, Z. Preparation methods of chemically cross-linked poly (ethylene glycol) hydrogels. Chem. Propellants Polym. Mater. 2011, 9, 36-43.

29. Ekblad, T.; Bergström, G.; Ederth, T.; Conlan, S.L.; Mutton, R.; Clare, A.S.; Wang, S.; Liu, Y.; Zhao, Q.; D’Souza, F.; et al. Poly(ethylene glycol)-containing hydrogel surfaces for antifouling applications in marine and freshwater environments. Biomacromolecules 2008, 9, 2775-2783. [CrossRef]

30. Owens, D.K.; Wendt, R.C. Estimation of the surface free energy of polymers. J. Appl. Polym. Sci. 1969, 13, 1741-1747. [CrossRef]

31. Fleming, I. Spectroscopic Methods in Organic Chemistry; McGraw-Hill: New York, NY, USA, 1980. [CrossRef]

32. Ying, W. Preparation and Properties of Polystyrene/reduced Graphene Oxide Core- shell Microspheres. Fine Chem. 2018, 2, 181-186. [CrossRef]

33. Zhou, H.; Xiong, Y.Z.; Wang, T.F.; Zeng, J.-X.; Liu, H.; Jian, J.; Yuan, Z.Q.; Zhou, Z.H.; Zeng, L.W.; Liu, G.Q. Synthesis of Polyurethane Hydrogel and Polyurethane Thermoplastic Elastomer Composite Based Separation Membranes. J. Nanosci. Nanotechnol. 2020, 20, 900-908. [CrossRef]

34. Jiang, D.; Liu, Z.; Han, J.; Wu, X. A tough nanocomposite hydrogel for antifouling application with quaternized hyperbranched PEI nanoparticles crosslinking. RSC Adv. 2016, 6, 60530-60536. [CrossRef] 